\title{
INFLUENCE OF CALCULATION ACCURACY ON THE TIME AND RESULTS OF SOLVING THE INVERSE PROBLEM OF MAGNETOSTATIC NONDESTRUCTIVE TESTING. NEED OF PARALLEL COMPUTATIONS
}

\author{
A. N. Pechenkov*, V. E. Shcherbinin \\ M.N. Miheev Institute of Metal Physics of Ural Branch of Russian Academy of Sciences, 18 S. Kovalevskoy st., \\ Ekaterinburg, Russian Federation \\ *Corresponding author. E-mail: Pechenkov@imp.uran.ru; address for correspondence: 18, ul. S. Kovalevskoy, 620990, \\ Ekaterinburg, Russian Federation. Tel.: +7 3433783735
}

Examples of computer modeling of the inverse problem are given. It is shown that the solution of such problem for defects of arbitrary form demands computing resources which can be provided only high-performance multiprocessor systems.

Keywords: magnetostatics, inverse problem, computer modeling.

DOI: $10.17804 / 2410-9908.2015 .5 .022-030$

\section{References}

1. Pechenkov A.N., Shcherbinin V.E. On the Solution of the Inverse Problem of Magnetostatic Tomography. Russian Journal of Nondestructive Testing, 2009, vol. 45, iss. 3, pp. 176-190. DOI: $10.1134 / \mathrm{S} 106183090903005 \mathrm{X}$.

2. Kung S.Y., Whitehouse H.J. and Kailath T., eds. VLSI and Modern Signal Processing. N. J. 07632, Prentice-Hall, Inc., Englewood Cliffs, 1985.

3. Akimova E.N., Vasin V.V., Perestoronina G.Y., Timerkhanova L.Y., Martyshko P.S., Koksharov D.Y. On regular methods for solving the inverse gravity problems on massively parallel computing systems. Numerical Methods and Programming, 2007, vol. 8, sec. 1, pp. 103-112.

4. Akimova E.N., Gemaidinov D.V. Parallel algorithms for solving the inverse gravity problem and the distant communication between the MVS-1000 and the user. Numerical Methods and Programming, 2008, vol. 9, sec. 1, pp. 129-140. 
Подана в журнал: 18.09.2015 г.

УДК 620.179.14

DOI: $10.17804 / 2410-9908.2015 .5 .022-030$

\title{
ВЛИЯНИЕ ТОЧНОСТИ ВЫЧИСЛЕНИЙ НА ВРЕМЯ И РЕЗУЛЬТАТЫ РЕШЕНИЯ ОБРАТНОЙ ЗАДАЧИ МАГНИТОСТАТИЧЕСКОЙ ДЕФЕКТОСКОПИИ. НЕОБХОДИМОСТЬ ПАРАЛЛЕЛЬНЫХ ВЫЧИСЛЕНИЙ
}

\author{
А. Н. Печенков*, В. Е. Щербинин \\ Федеральное государственное бюджетное учреждение науки Институт физики металлов имени \\ М.Н. Михеева Уральского отделения Российской академии наук, ул. С. Ковалевской, 18, Екатеринбург, \\ Российская Федераиия \\ *Ответственный автор. Электронная почта: Pechenkov@imp.uran.ru; адрес для переписки: 620990, \\ ул. С. Ковалевской, 18, Екатеринбург, Российская Федерация. Телефон: +7 (343) 378-37-35
}

Приведены примеры компьютерного моделирования обратной задачи. Показано, что решение такой задачи для дефектов произвольной формы требует вычислительных ресурсов, которые могут быть обеспечены только высокопроизводительными многопроцессорными системами.

Ключевые слова: магнитостатика, обратная задача, компьютерное моделирование.

\section{1. Введение}

Оценки необходимой точности вычислений при решении обратной задачи магнитостатической дефектоскопии в общем виде, с дефектами произвольной формы, вряд ли возможно получить теоретически. Поэтому в данной работе приведены некоторые результаты компьютерного моделирования ряда дефектов (немагнитных включений) - по мере усложнения моделей.

\section{2. Постановка задачи}

Моделирование состояло из двух этапов. Сначала решалась прямая задача для каждой модели: расчёт объёмного распределения намагниченности в намагничиваемом изделии и расчёт внешнего поля, создаваемого этим изделием. На втором этапе решалась обратная задача: оценка объёмного распределения намагниченности в изделии по выборке из этого внешнего поля. Формулы и алгоритмы моделирования приведены в [1].

Следует отметить, что для данной работы, на рассчитанное внешнее поле не накладывались дополнительные шумы. Прежде чем бороться с шумами, необходимо иметь такой вычислительно - алгоритмический комплекс, который обеспечивает приемлемое по времени и достоверности результатов решение обратных задач без шумов.

Моделирование проводилось на персональном компьютере с довольно низкими вычислительными характеристиками. Для выполнения процедур оптимизации, которые необходимы при приведении рассчитываемого внешнего поля к заданному, использовались две программы. Первая программа основана на одном из стандартных алгоритмов поиска минимума функции. Она работает точно, но достаточно медленно. Вторая программа имеет эвристический характер, т.е. работает менее точно, но более быстро. Во второй программе было заложено ограничение - одинаковое значение магнитной восприимчивости для всех ячеек сетки, в которых не обнаружены дефекты.

Обе программы не использовали производных целевой функции, так как вычислить их аналитически было невозможно. Сопоставление результатов работы этих двух программ и позволяет сделать некоторые предварительные выводы о требованиях к вычислительному комплексу магнитостатического томографа. 
onen-aCCeSS journal

\section{3. Результаты и обсуждение}

Первый, самый простой пример, приведён на рис. 1, 2. На рис. 1 показана расчётная область, покрытая сеткой из 64 кубических ячеек, содержащая немагнитные включения. Центры ячеек с немагнитными включениями помечены звёздочками. Видно, что имеется два поверхностных канавочных дефекта, один угловой и один - внутренний. Стрелками показано распределение вектора намагниченности.

На рис. $2 a$, $б$ показана компонента внешнего поля $\mathrm{H}_{\mathrm{z}}$, создаваемого данным распределением намагниченности в плоскостях сверху и снизу расчётной области (на расстоянии 0,5 размера ячейки сетки от границ расчётной области). Внешнее намагничивающее поле во всех примерах было однородно и направлено по оси Z.

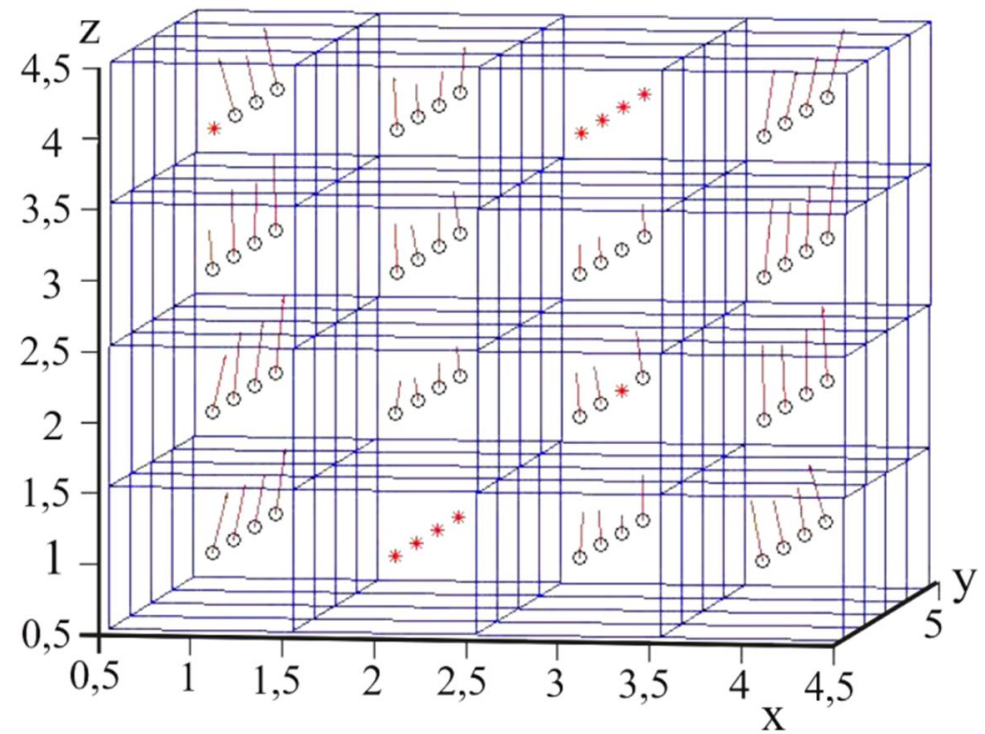

Рис. 1. Заданное расположение немагнитных включений в расчётной области
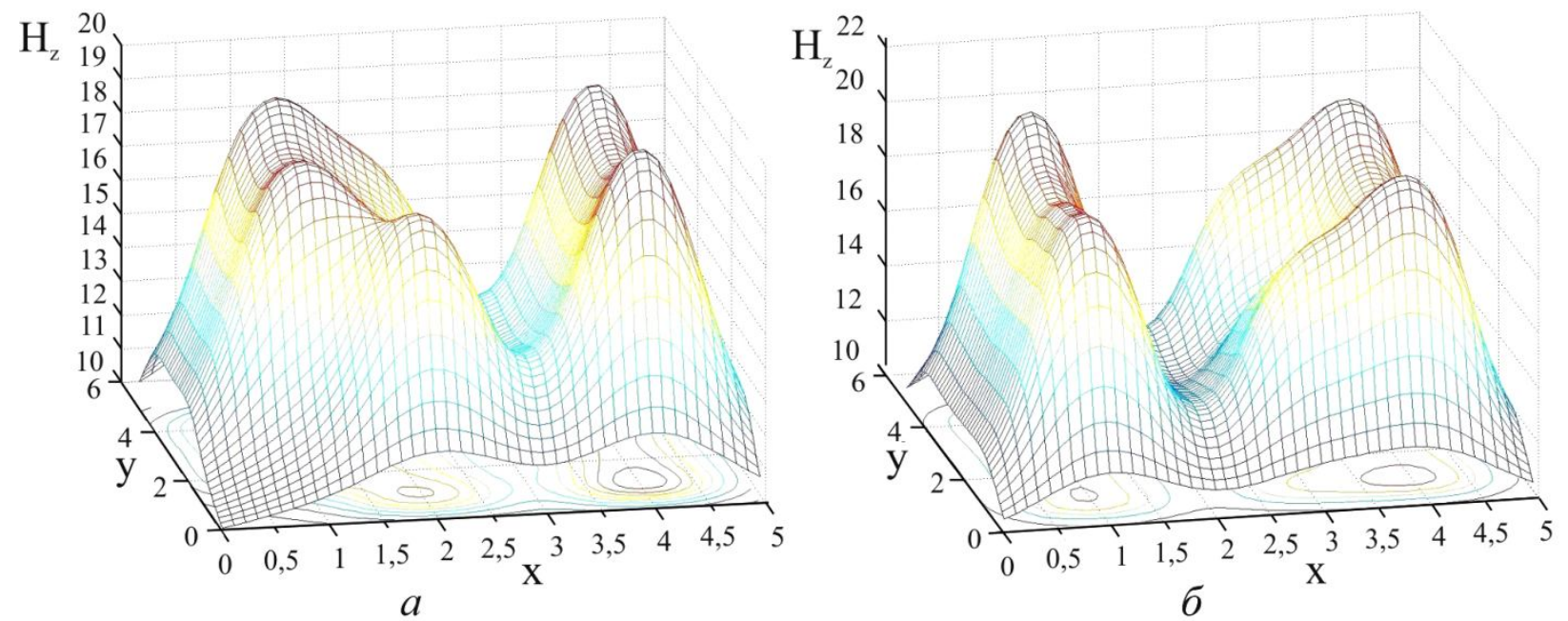

Рис.2. Компонента магнитного поля $\mathrm{H}_{\mathrm{z}}$ в плоскости: $a$ - над расчётной областью; $\sigma$ - под расчётной областью. Каждый график построен по выборке из 80 рассчитанных значений

По выборке из полей на рис. 2 обе упомянутые выше программы оптимизации правильно решили обратную задачу и определили положение немагнитных включений, показанных на рис. 1. 
Алгоритм со стандартной программой оптимизации затратил на решение этой задачи 3 ч 30 мин. Функция цели за первые 1 ч 45 мин была уменьшена примерно в 50 раз, с 0,71 до 0,015 (средняя невязка в точке измерения внешнего поля). Здесь не вычислялась относительная невязка в процентах, так как величины компонент поля сильно отличались друг от друга и лежали в диапазоне $0,03-10$.

При такой точности подгонки расчётного поля к заданному все дефекты определились правильно, кроме внутреннего дефекта, который оказался смещён на нижнюю плоскость сетки.

За вторые 1 ч 45 мин функция цели уменьшилась гораздо меньше - примерно в 3 раза, с 0,015 до 0,0047. После этого все дефекты определились точно, несмотря на серьёзный разброс вычисленных значений магнитной восприимчивости по ячейкам сетки $(65-165)$. Исходная магнитная восприимчивость была 80 (истинная - 100).

Время решения обратной задачи эвристическим алгоритмом составило 0,5 минуты, т. е. примерно в 420 раз быстрее, чем при использовании стандартной программы. При этом среднее значение функции невязки в точке измерения поля было уменьшено с 1,46 до 0,15. Значение магнитной восприимчивости в ячейках сетки без дефектов получилось 86,6 (исходная магнитная восприимчивость 80, истинная - 100). Правильное расположение дефектов получилось здесь при более низкой точности минимизации невязки. Вероятно, какую-то роль сыграло ограничение на значения магнитной восприимчивости в ячейках расчётной сетки. Но основная причина - все дефекты в данном примере поверхностные, кроме одного простого внутреннего дефекта. Поверхностные дефекты сильно влияют на топологию поля и поэтому легко идентифицируются при относительно низкой точности вычислений.

Таким образом, в обеих программах достигнутая точность минимизации функции невязки оказалась низкой, по сравнению с точностями $10^{-6}-10^{-8}$, желательными для вычислений на моделях без шумов. Достижение таких точностей не представляется возможным на упомянутом персональном компьютере.

Для следующего примера была взята расчётная сетка, содержащая 400 ячеек. Характер дефектов был оставлен таким же, как и в первом примере, но все эти дефекты были расположены в одном углу расчётной области, как показано на рис. 3. Остальная часть расчётной области была заполнена материалом изделия. На рис. 3, в отличие от рис. 1, не показаны векторы намагниченности в ячейках сетки, чтобы не загромождать изображение. На рис. 4 показана компонента поля $\mathrm{H}_{\mathrm{z}}$ в плоскостях сверху и снизу расчётной области.

Для этого и последующих примеров был использован только эвристический алгоритм минимизации невязки. Попытка применить стандартный алгоритм уже для этого примера, сетка которого содержит совсем немного ячеек, оказалась неудачной из-за неприемлемого времени расчётов. Так, на одно вычисление функции цели затрачивалось примерно в 30 раз больше времени, чем в первом примере. Кроме того, на порядок увеличивалось число вычислений функции цели, необходимое для проведения каждого шага процесса минимизации.

Эвристический алгоритм и в данном примере правильно решил обратную задачу и определил расположение дефектов в расчётной сетке. Однако время решения обратной задачи составило здесь 15 минут. При этом среднее значение функции невязки в точке измерения поля было уменьшено с 0,5 до 0,02. Значение магнитной восприимчивости в ячейках сетки без дефектов получилось 82 (исходная магнитная восприимчивость 80, истинная - 100). Правильное решение обратной задачи объясняется, по-видимому, простым характером дефектов. 


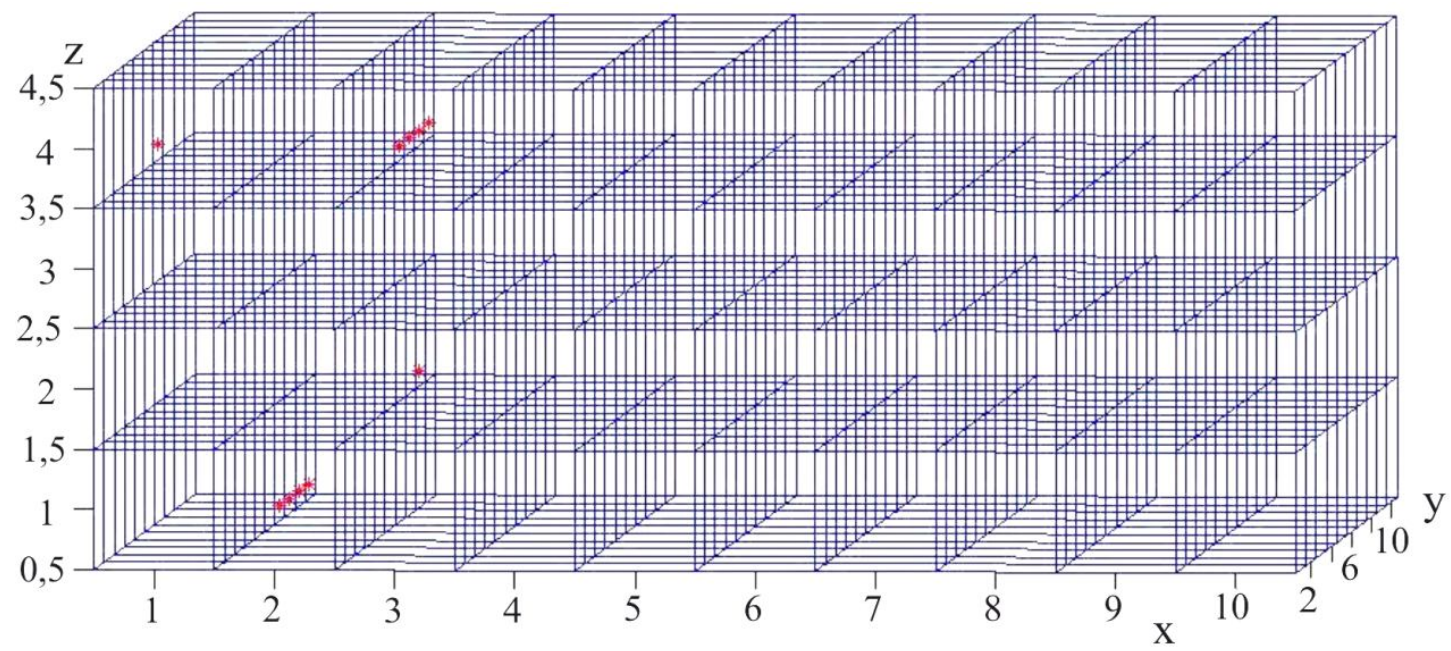

Рис. 3. Заданное расположение немагнитных включений в расчётной области
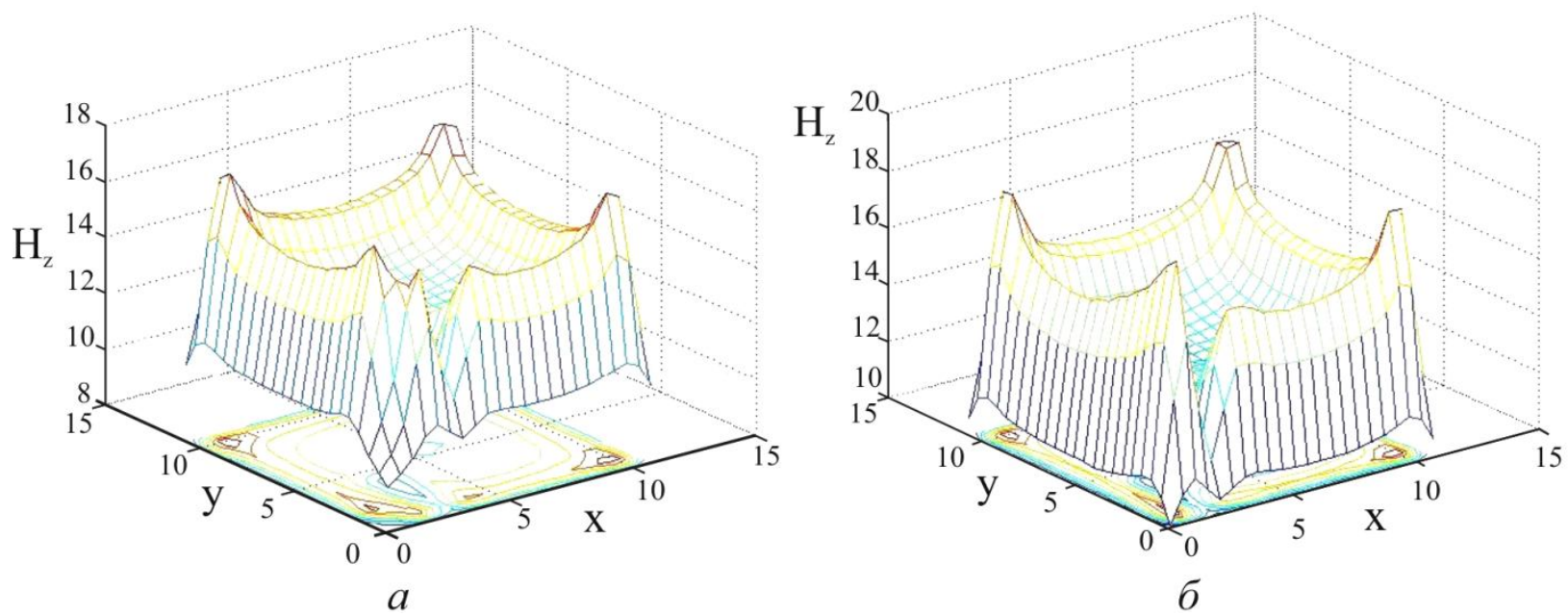

Рис. 4. Компонента магнитного поля $\mathrm{H}_{z}$ в плоскостях: $a$ - над расчётной областью; $\sigma$ - под расчётной областью. Каждый график построен по выборке из 400 рассчитанных значений

Следующий, третий, пример значительно усложнён. Расчётная область разбита на три слоя по вертикали. Каждый слой содержит 288 ячеек. Таким образом, вся расчётная область была разделена на 864 ячейки. Немагнитные включения заданы в каждом из трёх слоёв, как показано на рис. 5. Для того чтобы было легче ориентироваться в результатах расчетов, эти включения были выбраны в виде букв. Три буквы расположены в верхнем слое, две следующих буквы - во втором (внутреннем) слое и три буквы - в нижнем слое сетки. На рис. 6 показана компонента поля $\mathrm{H}_{\mathrm{z}}$ в плоскостях сверху и снизу расчётной области. 


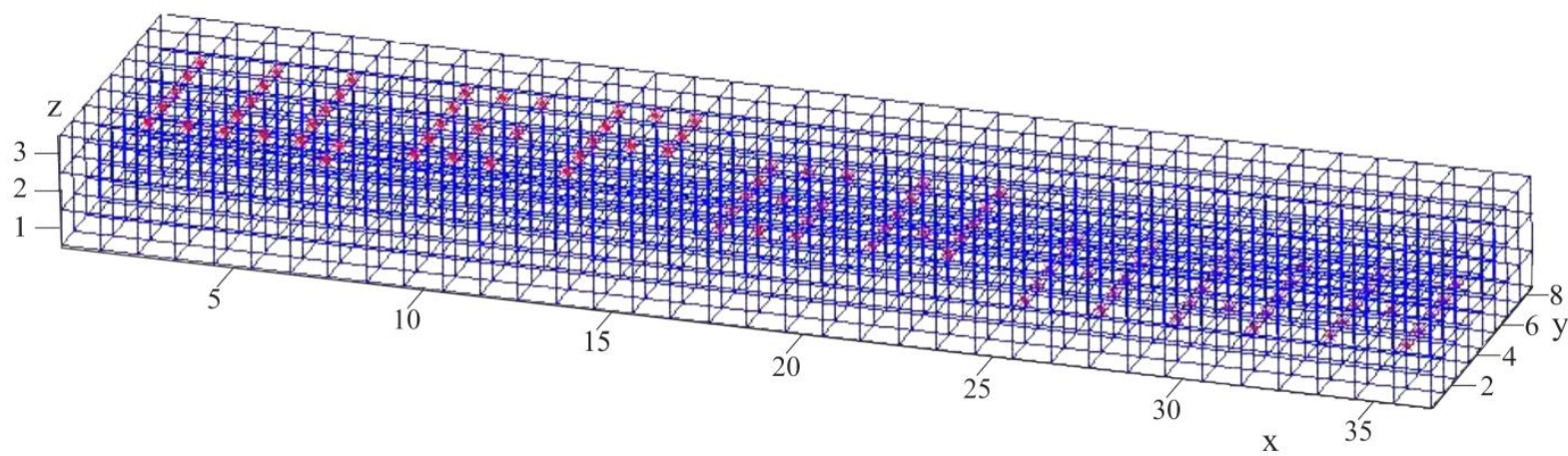

Рис. 5. Заданное расположение немагнитных включений в расчётной области
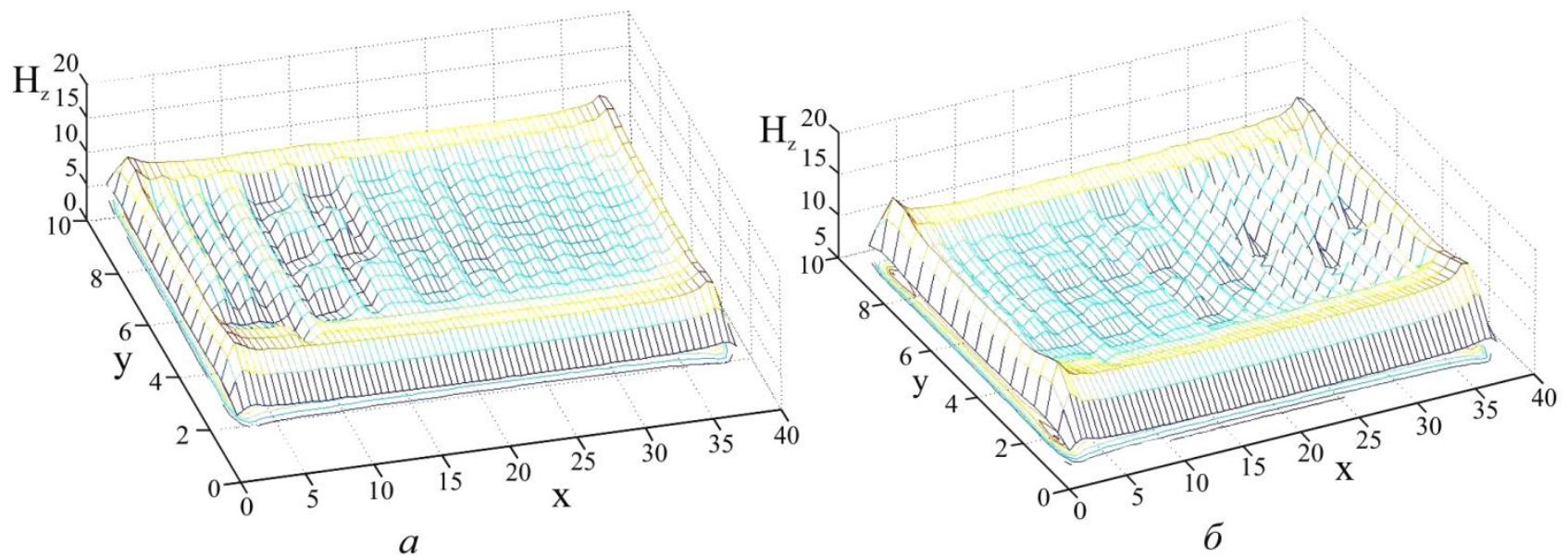

Рис. 6. Компонента магнитного поля $\mathrm{H}_{z}$ в плоскости; $a$ - над расчётной областью; $\sigma$ - под расчётной областью. Каждый график построен по выборке из 1050 рассчитанных значений
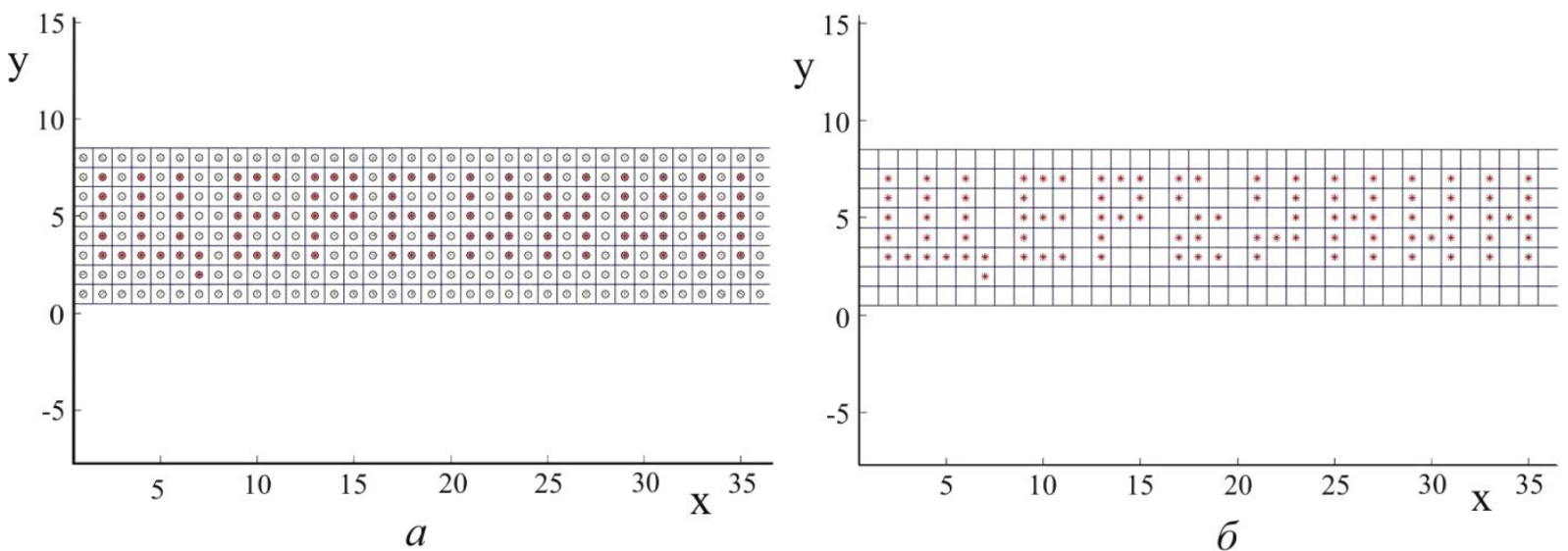

Рис. 7. Заданное $(a)$ и рассчитанное (б) расположение немагнитных включений в расчётной области. Вид сверху

Эвристический алгоритм в данном примере допустил 5 ошибок в расположении дефектов во внутреннем слое расчётной сетки. Чтобы легче увидеть эти ошибки, на рис. 7 приведён сквозной вид сверху всех дефектов в расчётной сетке. Время решения обратной задачи составило здесь 1 ч 50 мин. Нужно учитывать, что увеличилось не только число ячеек в сетке, но и число точек расчёта внешнего поля. Среднее значение функции невязки в точке измерения поля было уменьшено с 0,6 до 0,16. Значение магнитной восприимчивости в ячей- 
ках сетки без дефектов получилось 86,3 (исходная магнитная восприимчивость 80, истинная $-100)$.

Очевидно, что ошибки в данном примере вызваны недостаточной точностью эвристического алгоритма минимизации невязки.

Наконец, последний - четвёртый - пример, приведённый ниже, показывает полную неспособность эвристического алгоритма с недостаточной точностью минимизации невязки решить сложную обратную задачу, содержащую много слоёв внутренних дефектов (рис. 8).

В этом примере расчётная область состояла из 10 вертикальных слоёв, каждый из которых содержал 35 ячеек сетки. В каждом слое сетки опять содержался дефект в виде некоторой буквы. Таким образом, получалась очень сложная картина распределения дефектов по расчётной области.
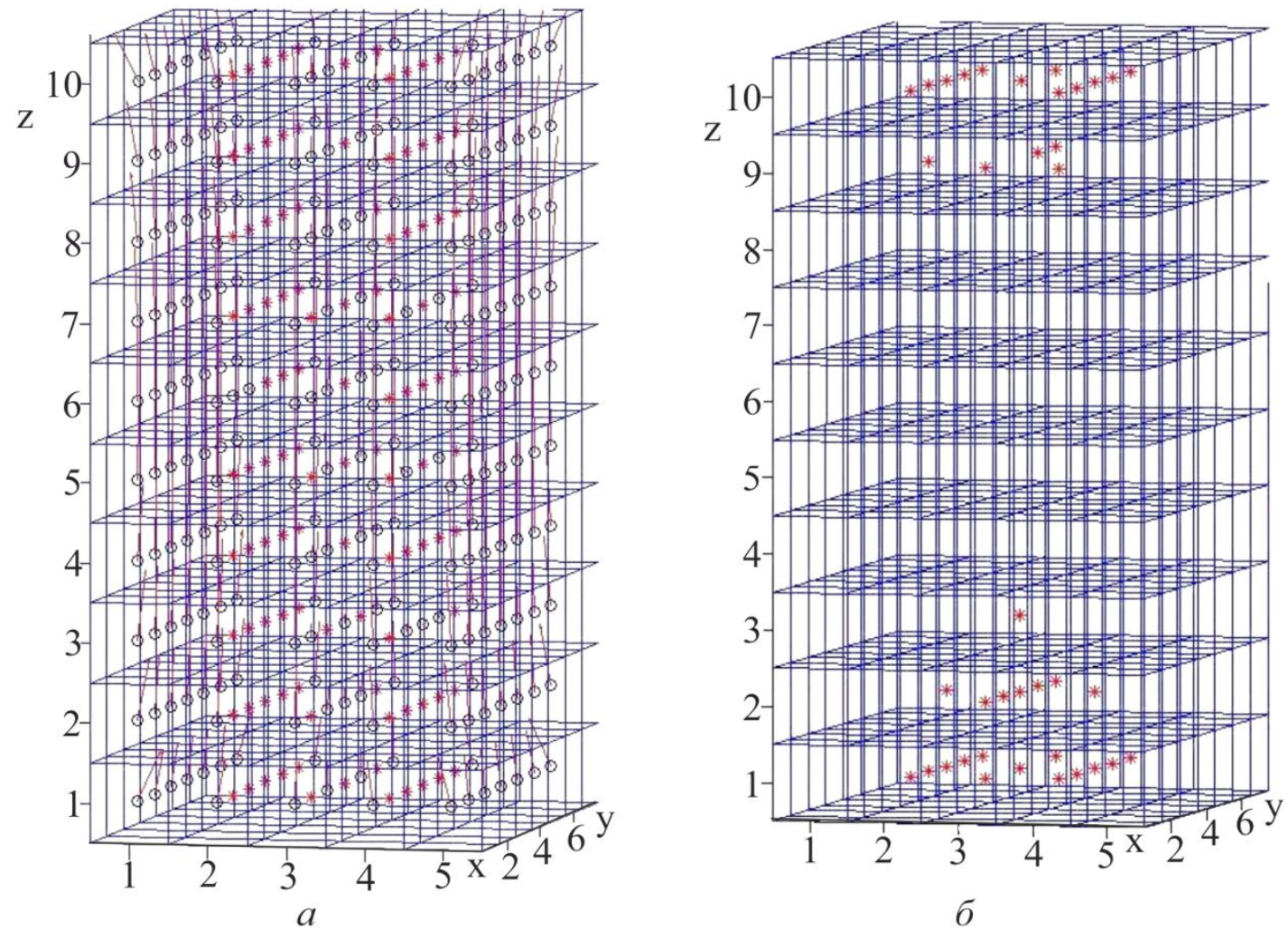

Рис. 8. Заданное и рассчитанное расположение немагнитных включений в расчётной области
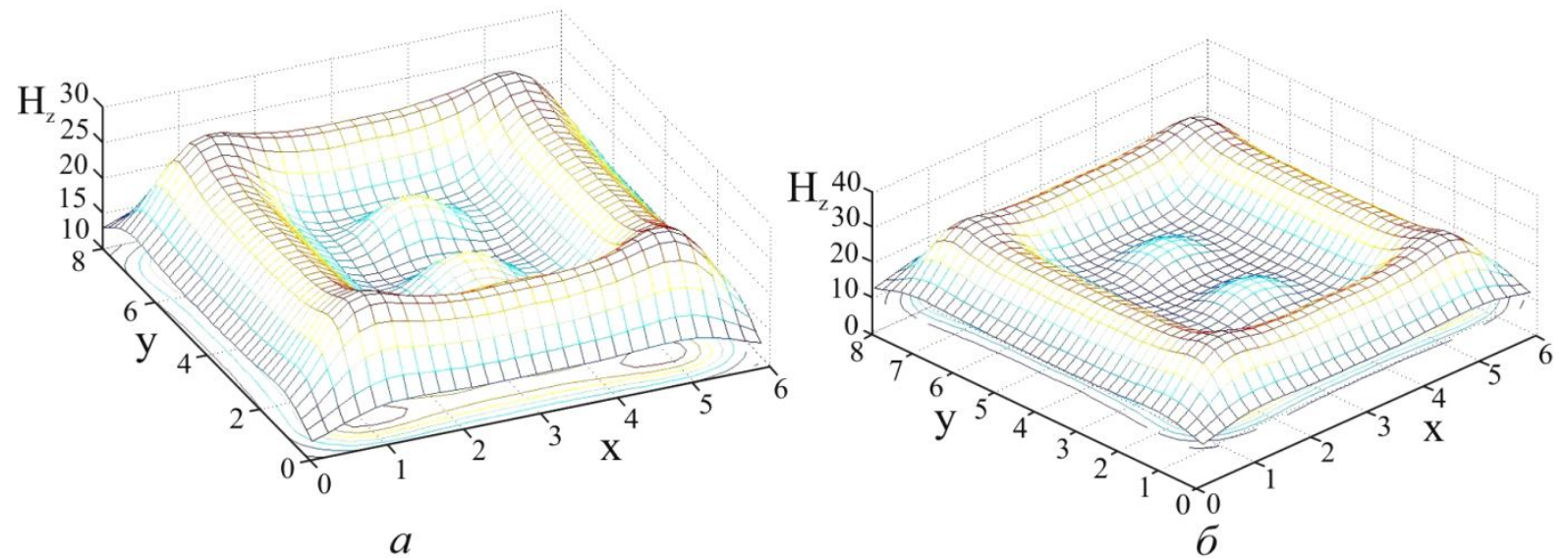

Рис. 9. Компонента магнитного поля $\mathrm{H}_{z}$ в плоскости: $a$ - над расчётной областью; $\sigma$ - под расчётной областью. Каждый график построен по выборке из 1050 рассчитанных значений 
Эвристический алгоритм в данном примере правильно определил только расположение дефектов в верхнем и нижнем поверхностных слоях расчётной сетки. Время решения обратной задачи составило здесь 30 минут. Среднее значение функции невязки в точке измерения поля было уменьшено мало: с 2 до 0,5. Значение магнитной восприимчивости в ячейках сетки без дефектов получилось 88,1 (исходная магнитная восприимчивость 80, истинная $-100)$.

Ясно, что внутренние ячейки сетки вносят очень слабый вклад во внешнее магнитное поле, который невозможно увидеть на рис. 9. Однако эти ячейки сильно влияют на намагниченность своих ближайших соседей и таким образом косвенно влияют и на величину внешнего поля изделия. Поэтому для решения таких обратных задач очень желательно пользоваться априорным материальным уравнением, позволяющим учитывать влияние ячеек друг на друга. При этом требуется не только очень точно минимизировать невязку на внешнем поле, но и точно решать прямую задачу, используя материальное уравнение. В данном примере точность вычислений была принесена в жертву скорости решения обратной задачи, что и привело к неудовлетворительному результату.

Следует отметить, что во всех примерах были правильно определены поверхностные дефекты, при малом времени и относительно невысокой точности вычислений. Это объясняется тем, что влияние этих дефектов на внешнее поле очень большое.

\section{4. Заключение}

Итак, время работы эвристического и стандартного алгоритмов минимизации в первом примере составило соответственно 0,5 мин и 210 мин. В третьем примере время работы эвристического алгоритма составило 110 мин., т. е. в 220 раз больше, чем в первом примере. Можно оценить (с большим занижением), что стандартный точный алгоритм работал бы $210 \times 220$ мин $\sim 32$ суток на этом примере. На более подробных сетках это время было бы во много раз больше.

Можно также предположить, что более мощный компьютер и более оптимальное программирование могли бы уменьшить эти времена в 10 - 20 раз. Однако этого всё равно не достаточно. Поэтому обратные задачи такого класса требуют для своего решения применения многопроцессорных вычислительных систем и методов параллельного программирования [2]. Параллельные алгоритмы вычислений в таких задачах основываются на том, что каждый процессор системы вычисляет поля, создаваемые группой ячеек сетки. Поэтому вычисления полей, создаваемых всеми группами, производится одновременно.

В процессе вычислений процессоры обмениваются результатами расчётов. Одновременность вычислений снижает время расчётов ещё примерно в $\mathrm{N}$ раз, где $\mathrm{N}$ - число процессоров в системе (обычно $\mathrm{N}=10-100)$.

Таким образом, для решения обратных задач магнитостатической дефектоскопии для дефектов произвольной формы требуется применение высокопроизводительных многопроцессорных вычислительных систем. Такой вывод согласуется с методами решения подобных обратных задач в других областях исследований, например в области обратных задач гравиметрии $[3,4]$. Так как математические постановки обратных задач в отмеченных и некоторых других областях очень похожи, то можно сказать, что для решения обратных задач определения тех или иных характеристик объекта по измерениям любого физического поля, созданного этим объектом, желательно создание многопроцессорного специализированного вычислительного комплекса с параллельной обработкой информации.

\section{Благодарность}

Работа выполнена по проекту фундаментальных исследований УрО РАН 2015-2017 гг., номер 15-17-2-54, «Компьютерное моделирование процесса функционирова- 
open-aCCess journal

ния магнитных сканеров на основе спинтроники для поиска и идентификации дефектов технологических трубопроводов компрессорных станций магистральных газопроводов»

\section{Литература}

1. Pechenkov A. N., Shcherbinin V. E. On the Solution of the Inverse Problem of Magnetostatic Tomography // Russian Journal of Nondestructive Testing. - 2009. - Vol. 45, iss. 3. - P. 176-190. - DOI: 10.1134/S106183090903005X.

2. VLSI and Modern Signal Processing / S. Y. Kung, H. J. Whitehouse and T. Kailath, eds. N. J. 07632 : Prentice-Hall, Inc., Englewood Cliffs, 1985.

3. On regular methods for solving the inverse gravity problems on massively parallel computing systems / E. N. Akimova, V. V. Vasin, Perestoronina, L. Y. Timerkhanova, P. S. Martyshko, D. Y. Koksharov // Numerical Methods and Programming. - 2007. - Vol. 8, sec. 1. - P. 103-112.

4. Akimova E. N., Gemaidinov D. V. Parallel algorithms for solving the inverse gravity problem and the distant communication between the MVS-1000 and the user // Numerical Methods and Programming. - 2008. - Vol. 9, sec. 1. - P. 129-140. 